\title{
Type 2 diabetes patients' preferences and willingness to pay for lifestyle programs: a discrete choice experiment
}

Jorien Veldwijk ${ }^{1,2^{*}}$, Mattijs S Lambooij ${ }^{1}$, Paul F van Gils ${ }^{1}$, Jeroen N Struijs ${ }^{1}$, Henriëtte A Smit ${ }^{2}$ and G Ardine de Wit ${ }^{1,2}$

\begin{abstract}
Background: Participation rates of lifestyle programs among type 2 diabetes mellitus (T2DM) patients are less than optimal around the globe. Whereas research shows notable delays in the development of the disease among lifestyle program participants. Very little is known about the relative importance of barriers for participation as well as the willingness of T2DM patients to pay for participation in such programs. The aim of this study was to identify the preferences of T2DM patients with regard to lifestyle programs and to calculate participants' willingness to pay (WTP) as well as to estimate the potential participation rates of lifestyle programs.
\end{abstract}

Methods: A Discrete Choice Experiment (DCE) questionnaire assessing five different lifestyle program attributes was distributed among 1250 Dutch adults aged 35-65 years with T2DM, 391 questionnaires (31\%) were returned and included in the analysis. The relative importance of the program attributes (i.e., meal plan, physical activity (PA) schedule, consultation structure, expected program outcome and out-of-pocket costs) was determined using panel-mixed logit models. Based on the retrieved attribute estimates, patients' WTP and potential participation rates were determined.

Results: The out-of-pocket costs $(\beta=-0.75, P<.001)$, consultation structure $(\beta=-0.46, P<.001)$ and expected outcome $(\beta=0.72, P<.001)$ were the most important factors for respondents when deciding whether to participate in a lifestyle program. Respondents were willing to pay €128 per year for individual instead of group consultation and $€ 97$ per year for 10 kilograms anticipated weight loss. Potential participation rates for different lifestyle-program scenarios ranged between $48.5 \%$ and $62.4 \%$.

Conclusions: When deciding whether to participate in a lifestyle program, T2DM patients are mostly driven by low levels of out-of-pocket costs. Thereafter, they prefer individual consultation and high levels of anticipated outcomes with respect to weight loss.

Keywords: Discrete choice experiment, Preferences, Diabetes mellitus type 2, Lifestyle program, Participation rate, Willingness to participate, Willingness to pay

\section{Background}

Participation rates of lifestyle programs among type 2 diabetes mellitus (T2DM) patients are less than optimal around the globe $[1,2]$. Yet, there appears to be notable delays in the development of the disease and the onset of diabetes-related complications among participants in

\footnotetext{
* Correspondence: Jorien.Veldwijk@rivm.nl

${ }^{1}$ National Institute for Public Health and the Environment (RIVM), Center for Nutrition, Prevention and Health Services, PO Box 1 (101), Bilthoven, BA 3720, The Netherlands

${ }^{2}$ University Medical Center Utrecht, Julius Center for Health Sciences and Primary Care, Utrecht, The Netherlands
}

\section{Biomed Central}

(c) 2013 Veldwijk et al.; licensee BioMed Central Ltd. This is an open access article distributed under the terms of the Creative Commons Attribution License (http://creativecommons.org/licenses/by/2.0), which permits unrestricted use, distribution, and reproduction in any medium, provided the original work is properly cited. different lifestyle programs [1-5]. Driven by these results, combined with the increasing prevalence of T2DM and high disease-specific mortality rates [6,7], lifestyle interventions have been included in standardized care protocols for the treatment of T2DM patients in several countries [8-10]. However, in general, the participation rates of such lifestyle programs among T2DM patients are unsatisfactory, ranging from $20-70 \%$ of those eligible for such programs $[1,2,11]$. Suboptimal participation rates are especially worrisome among the growing population of T2DM patients aged 35-65 years. Lifestyle changes 
among patients within this specific age group are expected to have a greater and more long-term impact on disease progress than among older T2DM patients [12]. Moreover, these relatively young T2DM patients suffer less from serious diabetes-related physical or medical restrictions $[13,14]$ or from other chronic diseases or disabilities $[15,16]$. Therefore, younger T2DM patients are relatively suitable candidates for participation in a lifestyle program.

In order to obtain a better insight into the motives of T2DM patients for participating in lifestyle interventions, various studies were conducted that resulted in an extensive list of barriers for participation in lifestyle interventions as reported by T2DM patients [17-21]. However, very little is known about the relative importance of such factors for these patients, while it seems reasonable to assume that not all of the factors are of equal importance in the decision-making process regarding the participation in a lifestyle program. Previous research has shown that this also applied with regard to lifestyle programs in other target populations [22-25].

A second omission in many studies on the willingness to participate in lifestyle programs is that the costs of lifestyle programs are often not included. If lifestyle interventions were to be implemented in 'real life', participants in such programs would have to pay at least part of the program costs out-of-pocket. Whereas participation in programs within a research setting tends to be free of charge. Taking into consideration that these programs need to be (partly) financed by the participants, it is worthwhile to examine the amount of money that potential participants would be willing to pay. Previous research among a non-diabetic population showed that the 582 individuals at a high risk of developing T2DM were willing to pay out-of-pocket for a lifestyle intervention [23]. These rates varied between $\$ 63$ and $\$ 5$ per month for a three-year course depending on the diet restrictions, hours of exercise, hour of counseling, use of medication, the goal that was set with respect to weight loss and the percentage expected reduction in T2DM risk [23].

Once we have obtained a better insight into and knowledge of program-related factors that are crucial for the participation of T2DM patients in these lifestyle programs as well as for their willingness to pay (WTP), recommendations can be made as to what type of program would most likely be preferred by its potential users. These recommendations can be taken into account when developing new lifestyle programs, thus increasing their reach, and hence their public health benefit.

The aim of this study was to identify the preferences of diabetes type 2 patients for different characteristics of lifestyle programs. Based on these preferences, participants' willingness to pay (WTP) as well as the potential participation rates of different lifestyle programs have been assessed.

\section{Methods}

\section{Participants and recruitment}

Within the Netherlands, generic diabetes care is arranged in care groups. A care group is a legal entity formed by multiple health care providers (however, these are often exclusively general practitioners (GPs)) [26]. A random selection of care groups (per province of the Netherlands) was contacted to distribute the questionnaire among T2DM patients aged 35-65 years who were not suffering from any serious diabetes-related complications (i.e., cardiovascular diseases, nephropathy, retinopathy, neuropathy) and who were registered with that care group. In total five care groups, located within five different provinces of the Netherlands, participated. These care groups distributed a total number of 1250 questionnaires to all the eligible patients within their care groups, 391 (31.3\%) of which were completed and included in the analysis. Due to confidentiality agreements with the care groups that distributed the questionnaires, no reminder letters could be sent. As a result, there was no non-response information available to empirically test whether responders differed from nonresponders with respect to their demographic and diseasespecific characteristics. The Dutch National Ethics Board (Central Committee on Research involving Human Subjects) concluded that formal testing by a medical ethical committee was not necessary, as T2DM patients were only required to complete an anonymous questionnaire once, which is in accordance with the guidelines laid down in the Declaration of Helsinki.

The mean age of the final study population was 57.2 years, more than half of the respondents were male (57.4\%), almost all respondents were Dutch nationals (94.1\%), and about half of the population (49.6\%) completed higher secondary education or lower general professional education (Coded 'medium' in Table 1). On average, the participants were diagnosed with T2DM 6.1 years ago and they had a mean $\mathrm{HbA}_{1 \mathrm{c}}$ of $49.9 \mathrm{mmol} / \mathrm{mol}$. Of all the respondents, $37.8 \%$ was overweight (indicated by a BMI between 25 and $30 \mathrm{~kg} / \mathrm{m}^{2}$ ) and $41.1 \%$ was obese (indicated by a BMI higher than $30 \mathrm{~kg} / \mathrm{m}^{2}$ ). The majority of the respondents reported no complications (78.3\%) and no other chronic conditions (99.7\%). 19\% of the respondents reported that they did not use any form of medication, $67.2 \%$ reported that they used glucose lowering medication in the form of pills, $4.6 \%$ reported that they injected insulin, and 9.2\% reported that they used both. Almost all respondents (94.1\%) reported that their primary health care contact was their GP and not a specialist in secondary care (e.g., an internist). Self-management measures were applied by approximately half of this population; $45.8 \%$ reported that they monitored their $\mathrm{HbA}_{1 \mathrm{c}}$ at home and $50.3 \%$ reported that they keep a T2DM diary.

In total $47.2 \%$ of this population reported that they considered lifestyle programs to be useful and $66.7 \%$ 


\begin{tabular}{|c|c|c|c|}
\hline & & Mean (SD) & Percentage \\
\hline Age $(n=385)$ & & $57.2(6.4)$ & \\
\hline Gender $(n=390)$ & Male & & 57.4 \\
\hline \multirow[t]{3}{*}{ Educational level $(n=379)$} & Low & & 31.1 \\
\hline & Medium & & 49.6 \\
\hline & High & & 19.2 \\
\hline Ethnicity $(n=390)$ & Dutch & & 94.1 \\
\hline Duration of diabetes (years) $(n=382)$ & & $6.1(5.5)$ & \\
\hline $\mathrm{HbA}_{1 \mathrm{c}}(\mathrm{mmol} / \mathrm{mol})(\mathrm{n}=101)$ & & $49.9(16.6)$ & \\
\hline Primary health care contact $(n=187)$ & GP & & 94.1 \\
\hline \multirow[t]{4}{*}{ Medication $(n=390)$} & None & & 19.0 \\
\hline & Oral glucose lowering medication & & 67.2 \\
\hline & Insulin & & 4.6 \\
\hline & Both & & 9.2 \\
\hline Complications present $(n=303)$ & & & 21.7 \\
\hline Chronic condition present $(n=390)$ & & & 0.3 \\
\hline \multirow[t]{4}{*}{ Weight category* $(n=364)$} & Underweight & & 0.2 \\
\hline & Normal weight & & 19.5 \\
\hline & Overweight & & 37.8 \\
\hline & Obese & & 41.1 \\
\hline EQ5d score $(n=391)$ & & $0.91(0.19)$ & \\
\hline \multirow[t]{2}{*}{ Self-management $(n=386)$} & monitoring $\mathrm{HbA}_{1 \mathrm{c}}$ at home & & 45.8 \\
\hline & Keeping a T2DM diary & & 50.3 \\
\hline \multirow[t]{2}{*}{ What is your opinion concerning lifestyle programs in general? $(n=388)$} & Very useful or useful & & 47.2 \\
\hline & Not useful at all & & 2.6 \\
\hline \multirow{2}{*}{$\begin{array}{l}\text { Do you think you are able to complete a lifestyle program that endures } \\
1 \text { year, without dropping out? }(n=391)\end{array}$} & Certainly or probably & & 33.5 \\
\hline & Certainly not & & 18.2 \\
\hline \multirow{2}{*}{$\begin{array}{l}\text { Would your partner, friends and/or family support you if you would } \\
\text { participate in a lifestyle program? }(n=391)\end{array}$} & Certainly or probably & & 66.7 \\
\hline & Certainly not & & 4.9 \\
\hline \multirow[t]{2}{*}{ Would you like to participate in a lifestyle program? $(n=391)$} & Certainly or probably & & 22.6 \\
\hline & Certainly not & & 19.4 \\
\hline
\end{tabular}

*Respondents were categorized as underweight if their BMI $<20 \mathrm{~kg} / \mathrm{m}^{2}$, normal weight if BMI $20-25 \mathrm{~kg} / \mathrm{m}^{2}$, overweight if BMI $25-30 \mathrm{~kg} / \mathrm{m}^{2}$ and obese if $\mathrm{BMI}>30 \mathrm{~kg} / \mathrm{m}^{2}$.

thought that their partner, family or friends would support their participation in a lifestyle program, 33.5\% felt that they would be able to complete such a lifestyle program and $22.6 \%$ would actually like to participate in such a program (Table 1).

\section{Discrete choice experiment}

DCEs are becoming a frequently used tool in public health research to estimate the average participation rates of interventions or medical treatments and to provide knowledge about the components of the programs that determine the participation rates $[27,28]$. The DCE methodology is based on the Random Utility Theory and assumes that any intervention or treatment can be described by its characteristics (i.e., attributes; such as frequency of consultation). The individual's preference for an intervention or treatment is dependent on the levels (e.g., weekly or monthly consultation) of those attributes $[27,28]$. By varying the levels of the attributes, different scenarios are constructed. Respondents are provided with at least two scenarios (i.e., choice tasks) simultaneously, they then have to choose the scenario that they prefer most. Each respondent is asked to complete a series of such choice tasks. In the end, conclusions can be drawn regarding the components that constitute an intervention that is most preferred by its potential users. 


\section{Attributes, levels and design}

The attributes and levels included in the current study were determined in a stepwise manner, which subsequently included a literature review, expert interviews and focus group interviews with T2DM patients. First, a list of barriers for participating in a lifestyle intervention by T2DM patients was compiled based on previously published literature [17-21]. Second, the list of barriers thus obtained was discussed during expert interviews with a physician, a dietician and a scientist with a specific interest in diabetes care. These expert interviews were conducted in order to [1] shorten the list of potential attributes and [2] to ensure that the attributes and levels were consistent with current practice. As a third step, four focus group interviews were conducted with 24 T2DM patients in order to ensure that [1] the most important attributes for the decision-making process of T2DM patients were included in the DCE and [2] proper levels were appointed to each of the attributes. Focus group interviews were conducted according to Krueger and colleagues [29]. This process led to the inclusion of five attributes (meal plan, physical activity (PA) schedule, consultation structure, expected outcomes and out-of-pocket costs) with three levels (Table 2).

Based on the selected attributes and levels, NGene 1.0 (ChoiceMetrics, 2011) software was used to develop a D-efficient design with 18 unique choice tasks [30,31]. To limit the burden of respondents, NGene divided these 18 choice tasks over two sets of nine choice tasks, each set of nine choice tasks was disseminated among half of the study population. Besides choosing one of the lifestyle programs presented, participants could also choose an optout solution. This opt-out option was included because, in real life, people can also choose not to participate in a lifestyle program. Table 3 presents an example of a choice task as included in the questionnaire of this study. Before completing these choice tasks, respondents were provided with an extensive explanation of the meaning of all attributes and levels as well as an explanation about how to deal with a choice task, accompanied by an example. Every choice task starts with the question: 'Imagine that your GP or nurse practitioner advises that you participate in a lifestyle program for a period of one year. In which situation would you prefer to participate, situation 1 or situation 2? If you do not wish to participate in either of the situations, you can tick the box "none". A questionnaire containing additional questions was added to the DCE, for further details on this questionnaire see Additional file 1.

The draft questionnaire was pilot tested among a subgroup $(n=20)$ of the study population to ensure that the wording used in the questionnaire was correct and understood by the target population. Most of the pilot tests were performed by means of a postal questionnaire, respondents were requested to mark every question or answering category that they did not understand or found hard to grasp and they were asked to provide suggestions for improvement. Moreover, three think aloud pilot tests were conducted to obtain more insight into how the respondents approached answering the choice tasks. No changes in the attributes and/or levels were deemed necessary based on the results of this pilot study. Moreover, based on the pilot-test data, sample size calculations were performed to ensure that significant differences for each attribute could be detected at a 5\% level [30,32].

\section{Statistical analyses}

NLogit 4.0 (Econometric Software, 2007) was used to construct the panel-mixed-logit (Panel-MIXL) models that were estimated within this study. When using such

Table 2 The attributes and levels that were included in this discrete choice experiment

\begin{tabular}{lccl}
\hline Attributes & Level 1 & Level 2 & Level 3 \\
\hline Meal plan: a plan, which describes the aims of the participants with respect to improvements in their diet, developed by the participants
\end{tabular}
of the program together with a coach

$$
\begin{array}{lll}
\text { Flexible: primarily based on the } & \text { General: includes general information on a } & \text { Elaborate: a patient tailored schedule that is } \\
\text { participants' own initiatives and ideas } & \text { healthy diet and provides example recipes } & \text { completely prepared by the lifestyle coach }
\end{array}
$$

Physical activity (PA) schedule: a plan, which describes the aims of the participants with respect to improvements in their PA behavior, developed by the participants of the program together with a coach

$$
\begin{array}{lll}
\text { Flexible: primarily based on the } & \text { General: includes general information on } & \text { Elaborate: a patient tailored schedule that is } \\
\text { participants' own initiatives and ideas } & \text { PA, and provides example exercises } & \text { completely prepared by the lifestyle coach }
\end{array}
$$

Consultation structure: the composition of the consults with the coach
Individually
Groups with 5 other T2DM patients
Groups with 10 other T2DM patients

Expected outcomes: the results, in terms of weight loss and physical fitness expected by the respondent after completion of a lifestyle program

$$
\begin{array}{ll}
\begin{array}{l}
\text { No weight loss but feeling more } \\
\text { healthy }
\end{array} & 5 \text { kilograms of weight lost and feeling } \\
\text { more healthy }
\end{array}
$$

10 kilograms of weight lost and feeling more healthy

Out-of-pocket costs: patients may have to pay (part) of the program costs out-of-pocket
75 euro per year
150 euro per year
225 euro per year 
Table 3 Example of a choice task

\begin{tabular}{lll}
\hline & Situation $\mathbf{1}$ & Situation $\mathbf{2}$ \\
\hline Peal plan & Flexible & General \\
Consulcal activity schedule & General & Elaborate \\
Expected outcome & Individual & In groups of 5 patients \\
& $\begin{array}{l}5 \mathrm{~kg} \text { weight loss and feeling } \\
\text { more healthy }\end{array}$ & $\begin{array}{l}10 \mathrm{~kg} \text { weight loss and feeling more } \\
\text { healthy }\end{array}$ \\
Tick the box of the situation that you prefer: & 0 & 150 euro per year \\
\hline
\end{tabular}

models, results are adjusted for the panel structure (i.e., multilevel structure) of the data. As every respondent completed nine choice tasks, their answers may be correlated, which is then accounted for. Equation 1 was tested using these models.

$\mathrm{U}=\mathrm{V}+\varepsilon=\beta_{0}+\beta_{1} *$ flexible meal plan $+\beta_{2} *$ elaborate meal plan

$+\beta_{3}$ flexible PA schedule $+\beta_{4} *$ elaborate PA schedule

$+\beta_{5} *$ consultation in groups of $5+\beta_{6} *$ consultation in groups of 10

$+\beta_{7} *$ expected outcome $+\beta_{8} *$ out of pocket costs $+\varepsilon$

$\mathrm{V}$ describes the measurable utility of a specific lifestyle program based on the attributes that were included in the DCE. $\beta_{0}$ represents the alternative specific constant and $\beta_{1}-\beta_{8}$ are the attribute estimates that indicate the relative importance of each attribute. Based on the model fit (AIC and Chi-square tests), the constant and the expected outcome attributes were set as random parameters (both with a normal distribution).

All nonlinear parameters were recorded into effect codes. In contrast to dummy coding, this coding procedure codes the reference category -1 , so the sum of the effect coded attributes is always 0 . The coefficient for the reference category is therefore $-1 *\left(\beta_{\text {effect code } 1}+\beta_{\text {effect code } 2)}\right)[33,34]$.

In order to calculate patients' marginal willingness to pay (WTP), the negative of the out-of-pocket attribute was used as a measure of the marginal utility of money. The ratio of either attribute estimate to this negative of the out-of-pocket attribute provides an estimation of patients' WTP concerning that specific attribute $[28,35]$. Moreover, the potential participation rates (choice probabilities) of a program that consists of a specific set of attributes was estimated. Since both the constant and the expected outcome attribute were included as random parameters in the analyses, choice probabilities could not be calculated directly, therefore a simulation was used $[28,33]$. The mean participation rates of all simulations $(n=1000)$ were estimated by taking the average of all simulated participation rate probabilities, which were calculated as $1 /\left(1+\exp ^{-v}\right)$.
For a more detailed description of the statistical methods used in this paper see Additional file 2.

\section{Results}

\section{Patient preferences}

Most of the attribute estimates were significant, indicating that they were important for T2DM patients when choosing whether to participate in a lifestyle program (Table 4). Participants did not have any distinct preferences with regard to the meal plan. However, they did prefer a general PA schedule above a flexible PA schedule. Participants reported a preference for individual consultation, as compared to consultation in groups of $10 \mathrm{pa}-$ tients. The greater the expected outcome in terms of weight loss, the more willing participants were to participate and higher out-of-pocket costs led to a decrease in their willingness to participate.

Since the magnitude of the beta values depends highly on the coding of the attributes, attributes were recorded into the same coding scale (all attribute levels were coded between -1 and 1) to enable the assessment of their relative importance. The results of the recoded analysis are not shown because they show a high degree of overlap with the results presented in Table 4 and provide betas that are difficult to interpret especially concerning the outcome and costs attributes. The results reveal that, based on the value of its coefficient, the out-of-pocket costs were the most decisive factor for respondents in determining whether they wanted to participate in a lifestyle program. This attribute was followed by the consultation structure and expected outcome. The least important factor in the decision-making process was the operationalization of the PA schedule.

The significant coefficient of the standard deviation of the expected outcome attribute indicates that there is indeed a high preference heterogeneity among respondents concerning the amount of weight loss they anticipate before starting the intervention.

Finally, the opt-out option was chosen in $46.3 \%$ of the choice tasks and $23.5 \%$ of the respondents chose to opt- 
Table 4 T2DM patients' preferences for a lifestyle program: the attribute estimates of the Panel-MIXL model

\begin{tabular}{|c|c|c|c|}
\hline Attribute & & Beta value & SE \\
\hline \multirow[t]{2}{*}{ Constant } & Mean & 0.11 & 0.13 \\
\hline & Standard deviation & $2.61^{*}$ & 0.71 \\
\hline \multirow[t]{3}{*}{ Meal plan } & Flexible & 0.11 & 0.07 \\
\hline & General (ref) & -0.04 & 0.06 \\
\hline & Elaborate & -0.06 & 0.06 \\
\hline \multirow[t]{3}{*}{ PA schedule } & Flexible & $-0.13^{*}$ & 0.06 \\
\hline & General (ref) & 0.02 & 0.01 \\
\hline & Elaborate & 0.11 & 0.06 \\
\hline \multirow[t]{3}{*}{ Consultation structure } & Individual (ref) & 0.50 & 0.08 \\
\hline & Groups of 5 & -0.04 & 0.06 \\
\hline & Groups of 10 & $-0.46^{* * *}$ & 0.08 \\
\hline \multirow[t]{2}{*}{ Expected outcome (10 kg) } & Mean & $0.72^{* * *}$ & 0.16 \\
\hline & Standard deviation & $1.53^{*}$ & 0.51 \\
\hline Out-of-pocket costs (€100) & & $-0.75^{* * *}$ & 0.08 \\
\hline
\end{tabular}

*significant at $\mathrm{p}<.05 ;{ }^{* * *}$ significant at $\mathrm{p}<.001$.

out in every choice task. Patient preferences did not change when these latter responders were excluded from the analysis.

\section{Willingness to pay}

The WTP was calculated for the significant attributes only. The results show that respondents were willing to pay $€ 21.0$ (95\% CI: €11.3; €30.7) for a switch from a flexible to a general PA schedule. Respondents were willing to pay an extra $€ 127.8$ (95\% CI: $€ 106.0$; $€ 149.7)$ per year for a lifestyle program organized via individual consultation instead of consultation with a group of 10 other patients. Respondents were willing to pay $€ 96.8$ (95\% CI: $€ 85.2$; $€ 108.4)$ per year for $10 \mathrm{~kg}$ anticipated weight loss.

\section{Potential participation rate}

A lifestyle program that consists of all of the least preferred attribute levels (flexible meal plan, flexible PA schedule, consultation in groups of 10 , no weight loss) was set as the 'base' model. This program showed the lowest participation rate (48.5\%) (Table 5). The potential participation rate increased if this 'base' model was adapted to the identified patient preferences (change to 51.5\%-57.4\%). The most preferred program, which includes a flexible meal plan, general PA schedule, individual consultation and a $10 \mathrm{~kg}$ weight loss, resulted in an estimated potential participation rate of $62.4 \%$.

\section{Discussion}

Our research is the first to demonstrate the relative importance of the factors that affect T2DM patients' preferences for a lifestyle intervention program. Results showed that the out-of-pocket costs were the most crucial factor
Table 5 Expected participation rates for different lifestyle programs based on the attribute estimates of the PanelMIXL model

\begin{tabular}{|c|c|c|}
\hline & $\begin{array}{l}\text { Participation } \\
\text { rates }(\%)\end{array}$ & Explanation \\
\hline Base model & 48.5 & $\begin{array}{l}\text { A program with a general meal plan, } \\
\text { a flexible PA schedule, consultation in } \\
\text { groups of } 10 \text { and no weight loss }\end{array}$ \\
\hline PA schedule & 51.5 & Base model with a general PA schedule \\
\hline $\begin{array}{l}\text { Consultation } \\
\text { structure }\end{array}$ & 54.8 & $\begin{array}{l}\text { Base model with individual } \\
\text { consultation }\end{array}$ \\
\hline $\begin{array}{l}\text { Expected } \\
\text { outcome }\end{array}$ & 57.4 & Base model with a $10 \mathrm{~kg}$ weight loss \\
\hline $\begin{array}{l}\text { Preferred } \\
\text { program }\end{array}$ & 62.4 & $\begin{array}{l}\text { A program with a flexible meal plan, } \\
\text { a general PA schedule, individual } \\
\text { consultation and a } 10 \mathrm{~kg} \text { weight loss }\end{array}$ \\
\hline
\end{tabular}

for T2DM patients when deciding whether to participate in a lifestyle program (i.e., patients preferred lowest costs). Moreover, a lifestyle program with a general physical activity component, individual consultation and large expected outcomes in terms of weight loss was preferred. T2DM patients were willing to pay $€ 21, € 128$ and $€ 97$ per year respectively for a lifestyle program with these desired levels of the attributes (i.e., a general PA schedule, individual consultation, and $10 \mathrm{~kg}$ anticipated weight loss). Additionally, it was estimated that approximately $62 \%$ of the T2DM patients aged 35-65 years would participate in a lifestyle program with these preferred levels.

Though there is limited evidence regarding T2DM patients' preferences for a lifestyle program, the current findings are comparable to those of previously conducted DCEs among other target populations describing different lifestyle interventions. The study by Johnson [23] found that individuals at a high risk of developing T2DM preferred a lifestyle program that specified anticipated weight loss over a program that did not describe any anticipated weight loss. They also found that programs with restrictive diets were disliked, that some sort of physical activity component was preferred, and that the respondents were willing to pay up to approximately $\$ 63$ per month for a total of 36 months for participation in the lifestyle program they preferred most [23]. Roux and colleagues [25], as well as Owen and colleagues [24], reported that participants in a lifestyle program preferred personally oriented programs that included both a diet and some sort of PA component. The diet component in the program should not be too restrictive [24], and participants were willing to pay for participation [25].

Patients in the current study reported to be willing to pay up to $€ 97$ per year for every 10 kilograms of anticipated weight loss. Though this seems a promising argument to boost participation without significantly increasing costs, one could argue that, despite the linearity of the initial costs and expected outcome attributes, beyond a certain point, 
participants are no longer willing to pay an additional $€ 97$ for an increase of 10 kilograms of anticipated weight loss. This was also shown by Johnson and colleagues who demonstrated nonlinearity of the WTP [36].

Finally, results showed that a potential participation rate of $62 \%$ can be expected when attributes are operationalized in accordance with patient preferences. Current programs can be improved by organizing individual consultation and communicating clearly about the anticipated outcomes of the program (in terms of weight loss and degree of physical fitness of the participant). The participation rates found in this study show that T2DM patients are willing to participate if programs meet certain criteria. However, the participation rates do not exceed the most optimistic participation rates currently observed in lifestyle programs $[1,2,11]$. This suggests that T2DM patients have additional motives for not participating in a lifestyle program other than the tested characteristics of a lifestyle program. These motives may differ considerably between individuals; previous research already suggested tailored lifestyle programs to enhance patient commitment $[37,38]$. Tailoring such programs to individual patients can be costly and less feasible compared to generic programs. It should be explored which factors, other than consultation structure and clear communication with respect to expected outcomes, can be maintained over the total target population in order to limit the variation between programs and to keep costs as low as possible while at the same time increasing patient commitment.

Our conclusions are restricted by a number of limitations. The usable response rate was $31.3 \%$. As the current study was questionnaire-based and participation was on a voluntary basis, selective non-response seems plausible. For instance, the number of non-Dutch patients was relatively low. This may be due to language difficulties, as a good command of the Dutch language is needed to complete the questionnaire and especially the DCE tasks. Therefore, generalizability with regard to preferences of non-Dutch patient groups remains limited. Besides, it could be the case that patients who already perceive their lifestyle as being healthy chose not to participate in this study. However, in real life, it is not likely that these patients would participate in a lifestyle program and therefore these patients are of limited interest for this specific study.

The current study included T2DM patients in the age group of 35-65 years, who do not suffer from severe diabetes-related complications. There is limited information on the representativeness of the current study population compared to the target population. Additional analyses of T2DM patients aged 35-65 in a large Dutch cohort study (EPIC-NL [39]) showed the same mean BMI values, but other characteristics could not be compared because of the specific inclusion and exclusion criteria of the current study (in particular, the exclusion of patients with severe diabetes-related complications in this study). Future research should be conducted among T2DM patients aged $>65$ years without any restrictions with respect to the presence of diabetes-related complications to obtain more insight into the preferences of this subpopulation. Researchers should then take into account that the attributes of the current DCE might not be applicable within this new target population. Conducting such research would contribute to the insights into (the differences and similarities in) preferences of the entire T2DM patient population with respect to lifestyle programs.

\section{Conclusions}

In conclusion, when deciding to participate in a lifestyle program, T2DM patients in the age group of 35-65 years are mostly driven by the out-of-pocket costs of a lifestyle program, the structure of the consultation and the expected outcome of the program. We therefore advise that lifestyle programs directed at T2DM patients should be set up based on individual consultation, while communicating about the expected outcomes of the program (in terms of weight loss) and keeping out-of-pocket costs as limited as possible.

\section{Additional files}

\section{Additional file 1: Detailed description of the additional}

questionnaire. Here a detailed description is provided about the

content of the questionnaire that was distributed alongside the DCE questionnaire.

Additional file 2: Detailed description of the statistical methods. Here a detailed description is provided about statistical background end methods that were used for the analysis in this study.

\section{Competing interests}

This research was funded by the Strategic Research fund of the National Institute of Public Health and the Environment. Sponsors were not part of the research group and therefore were not involved in conducting the research, writing the manuscript or submitting the final paper. None of the authors have stated that any competing interest exists.

\section{Authors' contributions}

$\mathrm{J}$ prepared the questionnaire, conducted the expert interview and focus groups, designed the DCE, performed the data analysis and wrote the manuscript. MSL contributed to all phases mentioned above. PFG and HAS were involved in writing the research protocol and have both critically reviewed the manuscript. JNS assisted in the recruitment of the care groups that distributed the questionnaire and critically reviewed the manuscript. GAW was the project leader of this research project and was therefore involved in all stages of this study. All authors read and approved the final manuscript.

Received: 3 June 2013 Accepted: 19 November 2013 Published: 29 November 2013

\section{References}

1. Knowler WC, Barrett-Connor E, Fowler SE, Hamman RF, Lachin JM, Walker EA, Nathan DM: Reduction in the incidence of type 2 diabetes with lifestyle intervention or metformin. N Engl J Med 2002, 346:393-403. 
2. Wing RR: Long-term effects of a lifestyle intervention on weight and cardiovascular risk factors in individuals with type 2 diabetes mellitus: four-year results of the Look AHEAD trial. Arch Intern Med 2010, 170:1566-1575.

3. Diabetes Prevention Program Research Group: The Diabetes Prevention Program (DPP): description of lifestyle intervention. Diabetes Care 2002, 25:2165-2171.

4. Lindstrom J, Eriksson JG, Valle TT, Aunola S, Cepaitis Z, Hakumaki M, Hamalainen $\mathrm{H}$, et al: Prevention of diabetes mellitus in subjects with impaired glucose tolerance in the Finnish Diabetes Prevention Study: results from a randomized clinical trial. J Am Soc Nephrol 2003, 14:S108-S113.

5. Aucott L, Gray D, Rothnie H, Thapa M, Waweru C: Effects of lifestyle interventions and long-term weight loss on lipid outcomes - a systematic review. Obes Rev 2011, 12:412-425.

6. Shaw JE, Sicree RA, Zimmet PZ: Global estimates of the prevalence of diabetes for 2010 and 2030. Diabetes Res Clin Pract 2010, 87:4-14.

7. Wild S, Roglic G, Green A, Sicree R, King H: Global prevalence of diabetes: estimates for the year 2000 and projections for 2030. Diabetes Care 2004 27:1047-1053.

8. American Diabetes Association: Standards of medical care in diabetes: 2010. Diabetes Care 2010, 33(Suppl 1):S11-S61

9. Paulweber B, Valensi P, Lindstrom J, Lalic NM, Greaves CJ, McKee M, Kissimova-Skarbek K, et al: A European evidence-based guideline for the prevention of type 2 diabetes. Horm Metab Res 2010, 42(Suppl 1):S3-S36.

10. IDF Clinical Guidelines Task Force: Global guideline for Type 2 diabetes. Brussels: International diabetes Federation; 2005.

11. James DVB, Johnston LH, Crone D, Sidford AH, Gidlow C, Morris C, Foster C: Factors associated with physical activity referral uptake and participation. J Sports Sci 2007, 26:217-224.

12. Bouchard DR, Langlois MF, Domingue MĖ, Brown C, LeBrun V, Baillargeon JP: Age differences in expectations and readiness regarding lifestyle modifications in individuals at high risk of diabetes. Arch Phys Med Rehabil 2012, 93:1059-1064.

13. Clarke PM, Gray AM, Briggs A, Farmer AJ, Fenn P, Stevens RJ, Matthews DR, et al: A model to estimate the lifestime health outcomes of patients with Type 2 diabetes: the United Kingdom Prospective Diabetes Study (UKPDS) Outcomes Model (UKPDS no. 68). Daibetologica 2004, 47:1747-1759.

14. Davies TME, Stratton IM, Fox CJ, Holman RR, Turner RC: U.K. Prospective Diabetes Study 22; Effect of age at diagnosis on diabetes tissue damage during the first 6 years of NIDDM. Diabetes Care 1997, 20:1435-1441.

15. van den Akker M, Buntinx F, Metsemakers JF, Roos S, Knottnerus JA: Multimorbidity in general practice: prevalence, incidence, and determinants of co-occurring chronic and recurrent diseases. J Clin Epidemiol 1998, 51:367-375.

16. van Oostrom SH, Picavet HS, van Gelder BM, Lemmens LC, Hoeymans $N$ van Dijk CE, Verheij RA, et al: Multimorbidity and comorbidity in the Dutch population - data from general practices. BMC Public Health 2012, 12:715-724.

17. Dube MC, Valois P, Prud'homme D, Weisnagel SJ, Lavoie C: Physical activity barriers in diabetes: development and validation of a new scale. Diabetes Res Clin Pract 2006, 72:20-27.

18. Forbes CC, Plotnikoff RC, Courneya KS, Boule NG: Physical activity preferences and type 2 diabetes: exploring demographic, cognitive, and behavioral differences. Diabetes Educ 2010, 36:801-815.

19. Lakerveld J, ljzelenberg W, van Tulder MW, Hellemans IM, Rauwerda JA, van Rossum AC, Seidell JC: Motives for (not) participating in a lifestyle intervention trial. BMC Med Res Methodol 2008, 8:17.

20. Thomas N, Alder E, Leese GP: Barriers to physical activity in patients with diabetes. Postgrad Med J 2004, 80:287-291.

21. Vijan S, Stuart NS, Fitzgerald JT, Ronis DL, Hayward RA, Slater S, Hofer TP: Barriers to following dietary recommendations in Type 2 diabetes. Diabet Med 2005, 22:32-38

22. Jendle J, Torffvit O, Ridderstrale M, Lammert M, Ericsson A, Bogelund M: Willingness to pay for health improvements associated with anti-diabetes treatments for people with type 2 diabetes. Curr Med Res Opin 2010, 26:917-923

23. Johnson FR, Manjunath R, Mansfield CA, Clayton LJ, Hoerger TJ, Zhang P: High-risk individuals' willingness to pay for diabetes risk-reduction programs. Diabetes Care 2006, 29:1351-1356.

24. Owen K, Pettman T, Haas M, Viney R, Misan G: Individual preferences for diet and exercise programmes: changes over a lifestyle intervention and their link with outcomes. Public Health Nutr 2010, 13:245-252.
25. Roux L, Ubach C, Donaldson C, Ryan M: Valuing the benefits of weight loss programs: an application of the discrete choice experiment. Obes Res 2004, 12:1342-1351.

26. Struijs JN, Baan CA: Integrating care through bundled payments-lessons from The Netherlands. N Engl J Med 2011, 364:990-991.

27. Lancsar E, Louviere J: Conducting discrete choice experiments to inform healthcare decision making: a user's guide. Pharmacoeconomics 2008, 26:661-677.

28. Ryan M, Gerard K, Amaya-Amaya M: Using Discrete Choice Experiments to Value Health and Health Care. Dordrecht: Springer; 2008.

29. Krueger RA, Casey MA: Focus groups; A practical guide for applied research. Thousand Oaks: Sage publications, Inc; 2000

30. Bliemer MCJ, Rose JM: Efficiency and sample size requirements for stated choice experiments. Washington DC: Transportaion Research Broad Annual Meeting; 2009

31. Huber J, Zwerina K: The Importance of Utility Balance in Efficient Choice Designs. J market res 1996, 33:307-317.

32. Marshall D, Bridges BF, Hauber B, Cameron R, Donnalley L, Fyie KFRJ: Conjoint analysis applications in health - how are studies being designed and reported?: an update on current practice in the published literature between 2005 and 2008. Patient 2010, 3:249-256.

33. Louviere JJ, Hensher DA, Swait JD: Stated Choice Methods; analysis and apllication. Cambridge: Cambridge University Press; 2000.

34. Bech M, Gyrd-Hansen D: Effects coding in discrete choice experiments. Health Econ 2005, 14:1079-1083.

35. Revelt D, Train KE: Mixed logit with repeated choices: households' choices of appliance efficiency level. Rev Econ Stat 1998, 80:647-657.

36. Johnson FR, Mohamed AF, Ozdemir S, Marshall DA, Phillips KA: How does cost matter in health-care discrete-choice experiments? Health Econ 2011, 20:323-330.

37. Resnicow K, Davis RE, Zhang G, Konkel J, Strecher VJ, Shaikh AR, Tolsma D, et al: Tailoring a fruit and vegetable intervention on novel motivational constructs: results of a randomized study. Ann Behav Med 2008, 35:159-169.

38. Williams DM, Papandonatos GD, Jennings EG, Napolitano MA, Lewis BA, Whiteley JA, Bock BC, et al: Does tailoring on additional theoretical constructs enhance the efficacy of a print-based physical activity promotion intervention? Health Psychol 2011, 30:432-441.

39. Beulens JW, Monninkhof EM, Verschuren WM, van der Schouw YT, Smit J, Ocke MC, Jansen EH, et al: Cohort profile: the EPIC-NL study. Int J Epidemiol 2010, 39:1170-1178.

doi:10.1186/1471-2458-13-1099

Cite this article as: Veldwijk et al:: Type 2 diabetes patients' preferences and willingness to pay for lifestyle programs: a discrete choice experiment. BMC Public Health 2013 13:1099.

\section{Submit your next manuscript to BioMed Central and take full advantage of:}

- Convenient online submission

- Thorough peer review

- No space constraints or color figure charges

- Immediate publication on acceptance

- Inclusion in PubMed, CAS, Scopus and Google Scholar

- Research which is freely available for redistribution

Submit your manuscript at www.biomedcentral.com/submit
C) Biomed Central 